\title{
Metabarcoding of the kombucha microbial community grown in different microenvironments
}

\author{
Oleg N Reva ${ }^{1 *}$, Iryna E Zaets², Leonid P Ovcharenko², Olga E Kukharenko², Switlana P Shpylova², \\ Olga $\vee$ Podolich², Jean-Pierre de Vera ${ }^{3}$ and Natalia O Kozyrovska ${ }^{2}$
}

\begin{abstract}
Introducing of the DNA metabarcoding analysis of probiotic microbial communities allowed getting insight into their functioning and establishing a better control on safety and efficacy of the probiotic communities. In this work the kombucha poly-microbial probiotic community was analysed to study its flexibility under different growth conditions. Environmental DNA sequencing revealed a complex and flexible composition of the kombucha microbial culture (KMC) constituting more bacterial and fungal organisms in addition to those found by cultural method. The community comprised bacterial and yeast components including cultured and uncultivable microorganisms. Culturing the KMC under different conditions revealed the core part of the community which included acetobacteria of two genera Komagataeibacter (former Gluconacetobacter) and Gluconobacter, and representatives of several yeast genera among which Brettanomyces/Dekkera and Pichia (including former Issatchenkia) were dominant. Herbaspirillum spp. and Halomonas spp., which previously had not been described in KMC, were found to be minor but permanent members of the community. The community composition was dependent on the growth conditions. The bacterial component of KMC was relatively stable, but may include additional member-lactobacilli. The yeast species composition was significantly variable. High-throughput sequencing showed complexity and variability of KMC that may affect the quality of the probiotic drink. It was hypothesized that the kombucha core community might recruit some environmental bacteria, particularly lactobacilli, which potentially may contribute to the fermentative capacity of the probiotic drink. As many KMC-associated microorganisms cannot be cultured out of the community, a robust control for community composition should be provided by using DNA metabarcoding.
\end{abstract}

Keywords: Kombucha microbial community, Metabarcoding, Pyrosequencing

\section{Introduction}

Culture-dependent methods have revealed an enormous microbial diversity in various fermented products. However, there is still much to be discovered about development and functioning of microbial communities. The high-throughput sequencing technologies known also as next generation sequencing (NGS) are in use to examine the phylogenetic diversity, composition, and

\footnotetext{
*Correspondence: oleg.reva@up.ac.za

${ }^{1}$ Bioinformatics and Computational Biology Unit, Department of Biochemistry, University of Pretoria, Lynnwood road, Hillcrest, Pretoria 0002, South Africa

Full list of author information is available at the end of the article
}

dynamic structural changes in microbial communities of fermented foods, giving an opportunity to describe and predict relationships between species in these complex ecosystems (Kim et al. 2011; Oguntoyinbo and Narbad 2012; Park et al. 2012; Nam et al. 2012a, b; Illeghems et al. 2012; Marsh et al. 2014). Applicability of NGS for metabarcoding and metagenomic analysis of environmental DNA samples allows identifying uncultured microbial species constituting the communities. Moreover, these approaches allow coupling of structural changes in the communities with environmental factors-e.g., temperature, salinity, $\mathrm{pH}$, etc.,--to perform a meta-analysis of dynamic changes of microbiota (Shade et al. 2013). DNA metabarcoding of complex bacterial and fungal

\section{Springer}


communities by profiling of $16 \mathrm{~S}$ rDNA sequences and internal transcribed regions (ITS) had opened new prospects in studying and designing of new efficient probiotics based on fermentation process.

Nowadays, when people became more concerned about obesity and prophylaxis chronic diseases, the probiotics and synbiotics have occupied an important sector within the functional food market. Most probiotic drinks are from dairy products. The tendency to veganism implied consuming of non-dairy nutraceuticals that called for design of new safe non-dairy probiotics, which became an essential health-keeping food category (Prado et al. 2008; Vasudha and Mishra 2013). Thus kombucha, in range with other fermented functional foods like kvass, fermented herb drinks, etc., may substitute dairy products for people with lactose intolerance (Gupta and Abu-Ghannam 2012). Fermented probiotic products are produced by complex microbial communities, which remain to be open environments characterized by rather unstable species composition dependent on nutritional sources and growth conditions.

Health improving effects of the kombucha probiotic beverage have been reported in a number of publications (Yapar et al. 2010; Bhattacharya et al. 2011, 2013; Aloulou et al. 2012; Kallel et al. 2012; Srihari et al. 2013). Kombucha microbial community (KMC) is an example of mutualistic metabolic cooperation of pro- and eukaryotic microorganisms (bacteria and yeasts). Several types of KMC are cultivated on different continents, which differed in the community structure and diversity (Teoh et al. 2004; Ovcharenko 2013; Marsh et al. 2014), but all of them always possessed cellulose-forming acetobacteria and yeasts. Close biochemical interplay between yeasts and bacteria was facilitated by enclosing the mixed community within cellulose-based pellicles created by the cellulose-producing bacteria on the surface of the liquid medium. The kombucha drink contains organic acids, amino acids, antibiotic substances, vitamins and also many other unidentified bioactive compounds beneficial for human health (Jayabalan et al. 2010). Kombucha was proved to exert an antimicrobial activity against pathogens (Battikh et al. 2012). Because of a relative stability of the community and the beneficial effect to human health, KCM was domesticated and widely spread around the world. It is usually cultivated in sweetened tea. Recently the kombucha and kombucha-like products with different supplements have been commercialized in many countries. It might be assumed that the KMC community is quite complex and many associated micro-organisms cannot be cultured out of the community. A robust control on the community composition might be provided by using NGS.
In this study, the microbial diversity of the kombucha variant from Ukraine (KMC-IMBG1) grown in different conditions was examined using both culture-dependent and culture-independent approaches. Study of a hybrid KMC-IMBG1 was performed to elucidate flexibility of $\mathrm{KMC}$ and its ability to recruit organisms from other communities in a similar way as it was reported for kimchi where additives had influenced the microbial community (Jung et al. 2011). Roche 454 pyrosequencing of amplified barcode sequences followed by a computer-based profiling of microbial species have uncovered multiple uncultivable members of KMC-IMBG1 in the pellicles and cultural liquid. KMC composition was depending on the growth conditions and showed ability to recruit accessory members such as lactobacilli.

\section{Materials and methods}

\section{Microbial cultures and culturing conditions}

The kombucha microbial culture was obtained from the collection of microorganisms of the Institute of Molecular Biology and Genetics of National Academy of Sciences (Kyiv, Ukraine). It was maintained in a filter sterilized black tea (Lipton, $1.2 \%$, w/v) extract with sucrose $(3.0 \%, \mathrm{w} / \mathrm{v})$ (sBTS) or non-sterile BTS (nsBTS). KMC-IMBG1 also has been maintained in filter $(0.22 \mu \mathrm{m}$, Millipore) sterilized black tea supplemented with honey $(2.0 \%)(\mathrm{BTH})$. A matured KMC-IMBG1 was obtained after cultivation for 14 days at $28^{\circ} \mathrm{C}$ without shaking. A hybrid KMC was obtained by growing KMC-IMBG1 in fermented cabbage brine. More specifically, the KMC cultural liquid (10\%), which previously was pre-cultured in $\mathrm{BTH}$, was added to the minced cabbage supplemented with honey $(2.0 \%)$. The cultivation conditions were the same as described above. Newly formed pellicles were used for inoculation of fresh BTH in a weekly basis for 5 weeks. For isolation and cultivation of acetobacteria, HS agar medium (Hestrin and Schramm 1954) was used. The isolates were incubated for 3-7 days at $30^{\circ} \mathrm{C}$ under stationary conditions in HS until formation of pellicles. Yeast cultures were isolated on the Glucose Yeast Peptone agar medium (HiMedia Laboratories, India). Concomitant bacteria were screened on the minimal agar medium with sucrose (Miller 1972). For medium selectivity, the antibiotics cycloheximide $(100 \mu \mathrm{g} / \mathrm{ml}$, SigmaAldrich) against yeast and ceftriaxone (50 $\mu \mathrm{g} / \mathrm{ml}$, Roshe) against bacteria were added to corresponding media.

\section{Confocal scanning laser microscopy}

After cultivation for 14 days in sBTS, the bacterial cellulose-based pellicle samples were fixed in formaldehyde vapor during $1 \mathrm{~h}$ and stained with calcofluor (excitation $405 \mathrm{~nm}$, filter BP 420-480) and thiazine red dyes 
(excitation $514 \mathrm{~nm}$, filter BP 530-600 nm). A microscopic examination of sample fluorescence was performed, using CSLM AXIOSKOP-2 ZEISS equipped with the LSM 510 PASCAL (CarlZeiss, FRG) software.

\section{DNA extraction}

Total DNA samples from the kombucha liquid culture and pellicle were isolated for further barcode amplification and pyrosequencing. Microbial DNA isolation from the 14 day-old KMC-IMBG1 liquid hybrid culture was performed with innuSPEED bacteria/fungi DNA isolation kit (Analytik Jena AG). In parallel, total DNA samples from cellulose-based hybrid kombucha pellicle (as well as the 14 day-old pellicles produced by KMCIMBG1 grown in sBTS, nsBTS, and BTH) were isolated from three specimens, using modified soft lyses method after blending of the pellicle (Gabor et al. 2003). The nucleic acids were quantified and qualified by a NanoDrop ND-1000 spectrophotometer (NanoDrop Technologies, Wilmington, DE).

\section{PCR amplification, DNA sequencing and analysis}

Bacterial and yeast isolates from KMC-IMBG1 were identified by PCR amplification using standard primers 27F/1494R (AGAGTTTGATCCTGGCTCAG/TGACTG ACTGAGGYTACCTTGTTACGACTT) for bacterial 16S rDNA and NL1/NL4 (GCATATCAATAAGCGGAGG AAAAG/GGTCCGTGTTTCAAGACGG) for fungal 26S rDNA amplification as it was described previously (Ogino et al. 2001; Kurtzman and Robnett 1997). More specifically, the PCR reactions for both primers were run for 35 cycles with annealing temperature $54^{\circ} \mathrm{C}$ for $27 \mathrm{~F} / 1494 \mathrm{R}$ and $52^{\circ} \mathrm{C}$ for NL1/NL4. PCR products were cleaned with UltraClean $^{\mathrm{TM}}$ PCR Clean-up DNA purification kit (MoBio Laboratories). The PCR products were sequenced by the Sanger method (Sanger et al. 1977) using Big Dye Terminator Sequencing Standard Kit v3.1 (Applied Biosystems, USA) and apparatus 3130 Genetic Analyser (Applied Biosystems). The $16 \mathrm{~S}$ rDNA sequences were binned by BLASTN search through the National Center for Biotechnology Information (NCBI) GenBank (US National Library of Medicine, Bethesda, Maryland, USA). These sequence data have been submitted to the GenBank database under an accession numbers KF908872-KF90879.

\section{DNA pyrosequencing}

DNA sequencing has been performed by using Roche GS FLX in Inqaba Biotec (http://www.inqababiotec.co.za). Pairs of standard primers 27F 5'-AGAGTTTGATCC TGGCTCAG-3' (Lane 1991) and 518R 5'ATTACC GCGGCTGCTGG-3' (Muyzer et al. 1993) for 16S; and ITS1 5'-TCCGTAGGTGAACCTGCGG-3' and ITS4
5'-TCCTCCGCTTATTGATATGC-3' (White et al. 1990) for ITS amplification were used. Generated 16S rRNA reads were checked for chimers by using DECIPHER algorithm (Wright et al. 2012) set for analysis of shortlength sequences. In total 30 putative chimeras were identified and removed from the read datasets. Quality control was performed by locally installed Fast QC program (http://www.bioinformatics.babraham.ac.uk/projects/fastqc). Poor quality reads with Phred quality score below 20 (that corresponded to $\mathrm{p}$ value $\geq 0.05$ ) and reads shorter than $100 \mathrm{bp}$ were filtered out.

\section{Metabarcoding dataset statistics}

DNA reads obtained from the sequencer were aligned by the local BLASTN against combined NCBI $16 \mathrm{~S}$ Microbial and GreenGenes16S databases for identification of $16 \mathrm{~S}$ rDNA reads and against the NCBI nt-database for identification of ITS reads. The latest versions of GreenGene and NCBI databases available at the time of running of this analysis, i.e., the mid of 2014, were used in this study. The BLASTN results were merged and visualized by MEGAN 5.2.3 (Huson et al. 2011). Additionally, the BLASTN output files were searched by an in-house BioPython based script to retrieve the statistics of the top scored hits over all reads. A taxon presence in a sample was accepted, if there were at least five reads binned to this taxonomic unit. The minimum BLASTN score for taxon identification was 300. Statistics of pyrosequencing is shown in Table 1.

Not filtered metabarcoding data sets were deposited in the Metagenomics RAST database server (4543580.3-4543590.3).

Expected species richness of a sample was estimated according to Chao 1 equation (Chao 1984):

$$
S_{\text {exp }}=S_{\text {obs }}+\frac{F_{1}^{2}}{2 F_{2}}
$$

where $S_{\text {exp }}$-expected species richness; $S_{\text {obs }}$-observed number of species; F1 is the number of singletons (i.e., the number of species with only a single occurrence in the sample) and F2 is the number of doubletons (the number of species with exactly two occurrences in the sample).

Rarefication curves were estimated by counting of number of identified species after successful binning of every 200 reads. An exception was the dataset ITS_sBTS when species number increment was measured every 100 successfully binned reads because of the small size of this dataset. The binning was considered as successful if the BLASTN score was $\geq 300$.

Distance between two metabarcode datasets was measured by the Eq. 2: 
Table 1 DNA reads obtained by Roche 454 sequencing of different samples

\begin{tabular}{|c|c|c|c|c|c|c|}
\hline Sample & $\begin{array}{l}\text { Total number of reads before and } \\
\text { after filtering and chimera removal }\end{array}$ & Total length, bp & Average & $\begin{array}{l}\text { Min. read } \\
\text { length }^{\mathrm{a}}\end{array}$ & $\begin{array}{l}\text { Max. read } \\
\text { length }\end{array}$ & $\mathrm{S}_{\mathrm{obs}} / \mathrm{S}_{\exp }^{\dagger}$ \\
\hline \multicolumn{7}{|l|}{ sBTS } \\
\hline Pellicle: $16 \mathrm{~S}$ & $2,384 / 2,356$ & $1,123,074$ & 471 & 77 & 607 & $14 / 46$ \\
\hline Pellicle: ITS & $532 / 530$ & 277,232 & 521 & 61 & 568 & $5 / 10$ \\
\hline \multicolumn{7}{|l|}{ BTH } \\
\hline Pellicle: $16 \mathrm{~S}$ & $2,632 / 2,626$ & $1,244,214$ & 472 & 47 & 828 & $14 / 46$ \\
\hline Pellicle: ITS & $7,888 / 7,783$ & $3,303,150$ & 418 & 43 & 561 & $23 / 87$ \\
\hline \multicolumn{7}{|l|}{ nsBTS } \\
\hline Pellicle: 165 & $1,880 / 1,828$ & 870,798 & 463 & 65 & 563 & $24 / 33$ \\
\hline Pellicle: ITS & $3,741 / 2,310$ & $1,138,925$ & 304 & 41 & 536 & $7 / 23$ \\
\hline \multicolumn{7}{|l|}{ Hybrid KMC } \\
\hline Pellicle: 165 & $8,716 / 8,250$ & $2,975,027$ & 341 & 40 & 513 & $9 / 10$ \\
\hline Pellicle: ITS & $7,943 / 7,113$ & $2,500,278$ & 314 & 40 & 541 & $18 / 34$ \\
\hline Liquid phase: $16 \mathrm{~S}$ & $6,494 / 6,325$ & $2,294,949$ & 353 & 40 & 513 & $16 / 17$ \\
\hline Liquid phase: ITS & $9,541 / 8,281$ & $3,165,774$ & 331 & 40 & 521 & $26 / 38$ \\
\hline
\end{tabular}

all reads shorter than $100 \mathrm{bp}$ were filtered out.

${ }^{\dagger} S_{\text {obs }}$ observed number of species including those identified by a single read, $S_{\text {exp }}$ expected number of species according to Chao estimation (Eq. 1).

$$
D=\sqrt{\frac{\sum_{\text {comb }}\left(m_{1} / N_{1}-m_{2} / N_{2}\right)^{2}}{N_{\text {comb }}}}
$$

where $\mathrm{N}_{\text {comb }}$-total number of identified species in both datasets; $\mathrm{m}_{1}$ and $\mathrm{m}_{2}$-numbers of reads binned to the species $m$ in the datasets 1 and 2, respectively; $N_{1}$ and $\mathrm{N}_{2}$-total numbers of binned reads in the datasets 1 and 2 , respectively. Distances were used to infer dendrograms of dataset diversity by using the Neighbor-joining algorithm implemented in MEGA6 (Tamura et al. 2013).

\section{Results}

Isolation of cultivable forms of microorganisms associated with KMC

DNA fragments amplified by PCR from DNA samples extracted from cultivable isolates of KMC-IMBG1 were binned to taxonomic units by BLASTN alignment. Members of four yeast genera Pichia, Brettanomyces/Dekkera, Candida and Zygosaccharomyces; and two bacterial genera Gluconacetobacter (now Komagataeibacter gen. nov., Yamada et al. 2012) and Gluconobacter were identified. On the species level there were Komagataeibacter sp. (99\% homology to K. xylinus and K. saccharivorans), K. intermedius, K. kombuchae, and Gluconobacter oxydans. As it was revealed by culture methods, the simplest structure of KMC cultivated in sterile black tea with sugar (sBTS) composed of two yeast species of Pichia and Brettanomyces/Dekkera; and two acetobacteria: Komagataeibacter sp. and K. intermedius. In classic non-sterile sweetened black tea medium (nsBTS), KMC-IMBG1 comprised Pichia sp., Dekkera anomala, Candida sp., Komagataeibacter sp., K. intermedius and Gluconobacter oxydans. Additional yeast species Zygosaccharomyces bailii and acetobacterium K. kombuchae were isolated from the culture maintained in the sterile black tea medium with honey (BTH). In the hybrid kombucha culture grown in BTH mixed with cabbage brine, several atypical bacterial species have been identified including Bacillus subtilis, B. pumilis (Firmicutes) and Microbacterium sp. (Actinobacteria).

At the same time, the confocal scanning laser microscopy revealed a higher level of diversity of KMC-IMBG1 especially those associated with the cellulose $3 \mathrm{D}$ web (Additional file 1: Figure S1). It was hypothesized that uncultivable microbial organisms might be abundant in this network. Particularly, there were peculiar long cells observed during the dormancy state (Additional file 1: Figure S1b), which were dissimilar to any cultivable bacteria (Puspita et al. 2012). To overcome the problem of identification of uncultivable representatives of KMCIMBG1, a metabarcoding approach has been used.

\section{Metabarcoding analysis of KMC-IMBG1 grown in different conditions}

According to the results of analysis of metabarcodes, the acetobacteria of Komagataeibacter and Gluconobacter genera ( $\alpha$-Proteobacteria) dominated in KMCIMBG1 grown in sBTS, nsBTS and BTH with a few other bacterial species of Komagataeibacter. K. xylinus prevailed in all analysed bacteriomes (77.7-96.8\%); $K$. intermedius reached up to $4.9 \%$, and Gluconobacter 
spp., most of which belonged to G. oxydans, composed up to $10 \%$ of bacterial community in BTH. However, when grown in nsBTS, the proportion of Gluconobacter decreased 50 -folds (Figure 1). This can be explained by the preferred consumption of different sugars present in honey (Mandal and Mandal 2011). Estimated richness of bacterial and fungal species of KMC is shown in Table 1. Interestingly, Gluconoacetobacter diazotrophicus known as an obligate sugarcane endophyte (Baldani et al. 1997) was constantly present in all variants of KMC studied in this work; however, this species was not isolated by culture-dependent method. Herbaspirillum spp. and Halomonas spp. were a minor, but permanent component in KMC-IMBG1 grown in all the different conditions. Presence of Halomonas sp. was also reported in kombucha microbiota revealed in the metabarcoding study by Shade (2011). The minor fractions of the KMC-IMBG1 were represented by several occasional Firmicutes, $\beta$-and $\gamma$-Proteobacteria (see Figure 1).

The metabarcoding showed that KMC-IMBG1 comprised yeast species belonging to Pichia, Brettanomyces/Dekkera, Candida and Saccharomyces genera, as well as unknown OTUs similar to 'compost fungus' and 'unknown yeast.' The yeast composition widely varied in different cultures. The major yeast species of KMCIMBG1 grown in sBTS was Dekkera anomala. Pichia fermentas was abundant in BTH, and Pichia occidentalis (former Issatchenkia occidentalis) was the most frequent

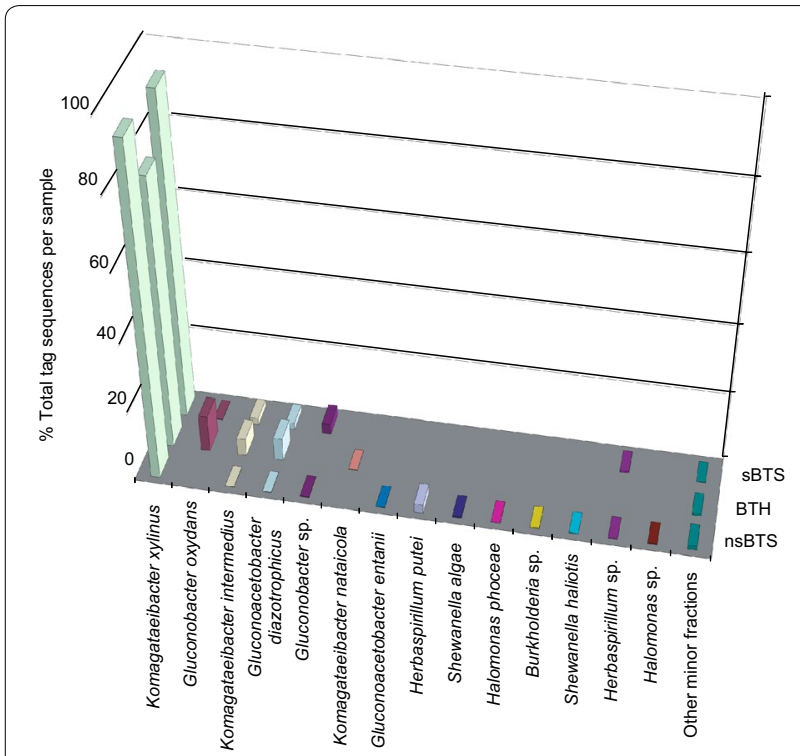

Figure 1 Profiles of bacterial species of KMC-IMBG1 grown in sterile black tea with sugar (sBTS), non-sterile black tea with honey (BTH) and non-sterile black tea with sugar (nsBTS) identified by binning of 165 rDNA reads. in nsBTS (Figure 2). This observation suggested that the domination of that or another yeast species significantly depended on the cultivation conditions at much higher extend than it was observed for the core bacterial community (see Figures 1,2). It was remarkable that an uncultured unknown fungal species identified as 'compost fungus' was the most abundant in $\mathrm{BTH}$ and to some extend in nsBTS.

\section{Metabarcoding analysis of the hybrid kombucha culture}

Bacterial and yeast communities of the hybrid KMCIMBG1 grown in a mixture of filter-sterilized BTH with added sweetened fermented cabbage brine were expectedly much more diverse (Figure 3 ). K. xylinus was a dominant bacterial species. Lactobacilli, which probably originated from the cabbage brine and remained here in series of passages, were abundant in the hybrid KMC-IMBG1 pellicles. Lactobacillus spp. isolates were reported before as indispensable kombucha community members (Marsh et al. 2014). Lactobacilli are known also as the indigenous inhabitants of fermented cabbage (Jung et al. 2011). L. plantarum causes fermentation of cabbage carbohydrates to the lactic acid or acetic acid.

Several bacterial OTUs failed with taxonomic affiliation because of lack of appropriate reference sequences in the searched databases (Figure 3a). Yeast DNA barcoding discovered a much higher number of OTUs in pellicles

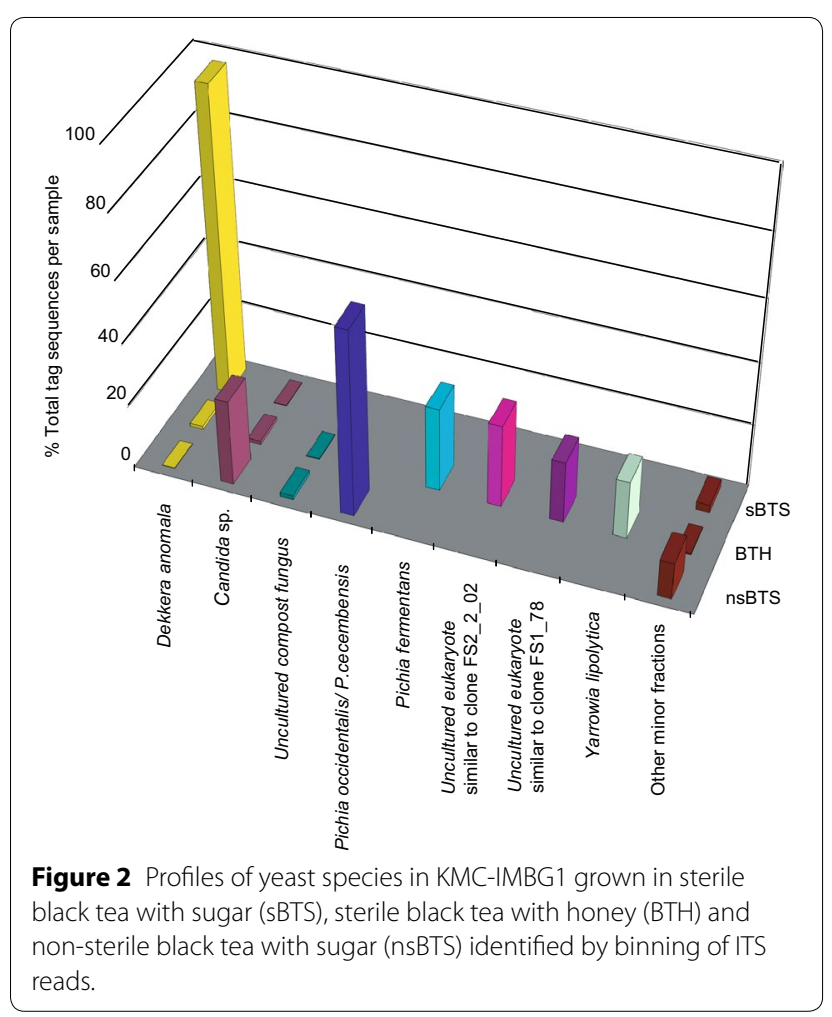




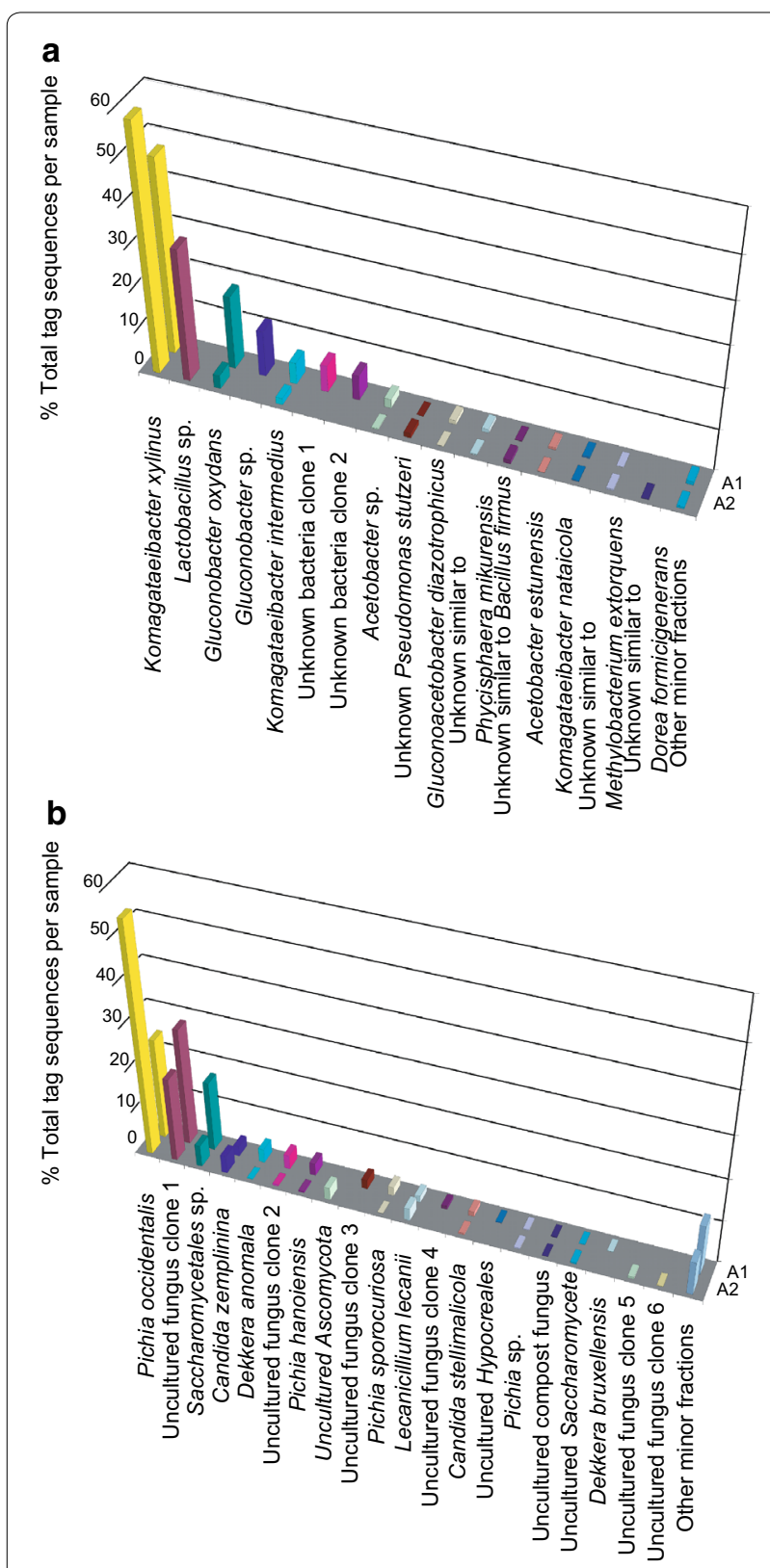

Figure 3 Normalized abundance of the most frequent OTUs of KMC identified by BLASTN in a $16 \mathrm{~S}$ rDNA and $\mathbf{b}$ ITS reads. A1 - liquid phase of the culture; A2 - cellulose based biofilm. Numbers of identified reads were normalized by the total numbers of reads in the samples.

and cultural liquid in the hybrid kombucha culture as compared to the parental KMC-IMBG1. P. occidentalis/P. cecembensis were the dominant yeast species the same as in nsBTS (Figure 3b). Many OTUs were not affiliated to any taxonomic units because of a weak sequence similarity, or they showed similarity to unknown microorganisms. Anyway, even the weak similarity was consistently against the same reference sequences that suggested that the total number of species in KMC-IMBG1 was limited but many of them still remained unknown.

\section{Comparative analysis of microbiomes produced by KMC under different microenvironments}

Rarefication curves for studied metabarcode datasets and dendrograms representing species diversity of KMC pellicle grown at different conditions are shown in Figure 4. Remarkably, fungal biomes of KMC varied to a much higher extend depending on the growth conditions than the bacterial component (see the scaling bars in dendrograms in Figure 4). The biggest number of bacterial OUTs, which were singletons or represented only by a few reads, was observed in KMC grown in non-sterile conditions (nsBTS). It is reflected in the steepness of the corresponding rarefication curve in Figure 4. Interestingly, the richness of fungal species of the sample was depleted, that may be explained by presence of Bacillus and Pseudomonas, which might synthesize antifungal antibiotics. Nevertheless, the bacterial core component in nsBTS remained the same. The biggest alteration in the KMC bacteriome structure was observed in BTH (Figure 4a) caused probably by honey addition. Microbial composition of the hybrid KMC including both the bacterial and fungal components showed a higher level of stability as the rarefication curves calculated for this community has got faster the saturation level (Figure 4c, d).

\section{Discussion}

This study showed that KMC-IMBG1 is quite flexible and variable community. The main highlight of this study was that KMC-IMBG1 grown on different sterile and non-sterile media produced a stable core microbiome comprising acetobacteria and few associated strains of yeast species, and a number of accessory species, which may or may not occur at different conditions. The core part of KMC-IMBG1 probably is critical for functioning of the whole community and might be responsible for recovery of the community after disturbance. In addition to prevalent community members, several minor but permanently occurring bacterial species of KMC-IMBG1 were also discovered. Among them there were Herbaspirillum spp. and Halomonas spp. These organisms were identified by binning the DNA reads originated from KMC grown in sterile and non-sterile media. In other studies on species composition of kombucha from North American and Ireland ecotypes these species were not reported (Marsh et al. 2014). Another disagreement with the report by Marsh et al. (2014) was that according to these authors the highest diversity of micro-flora was associated with the cellulose pellicle. In Figure $4 c, d$ it is seen in rarefication curves of the hybrid 


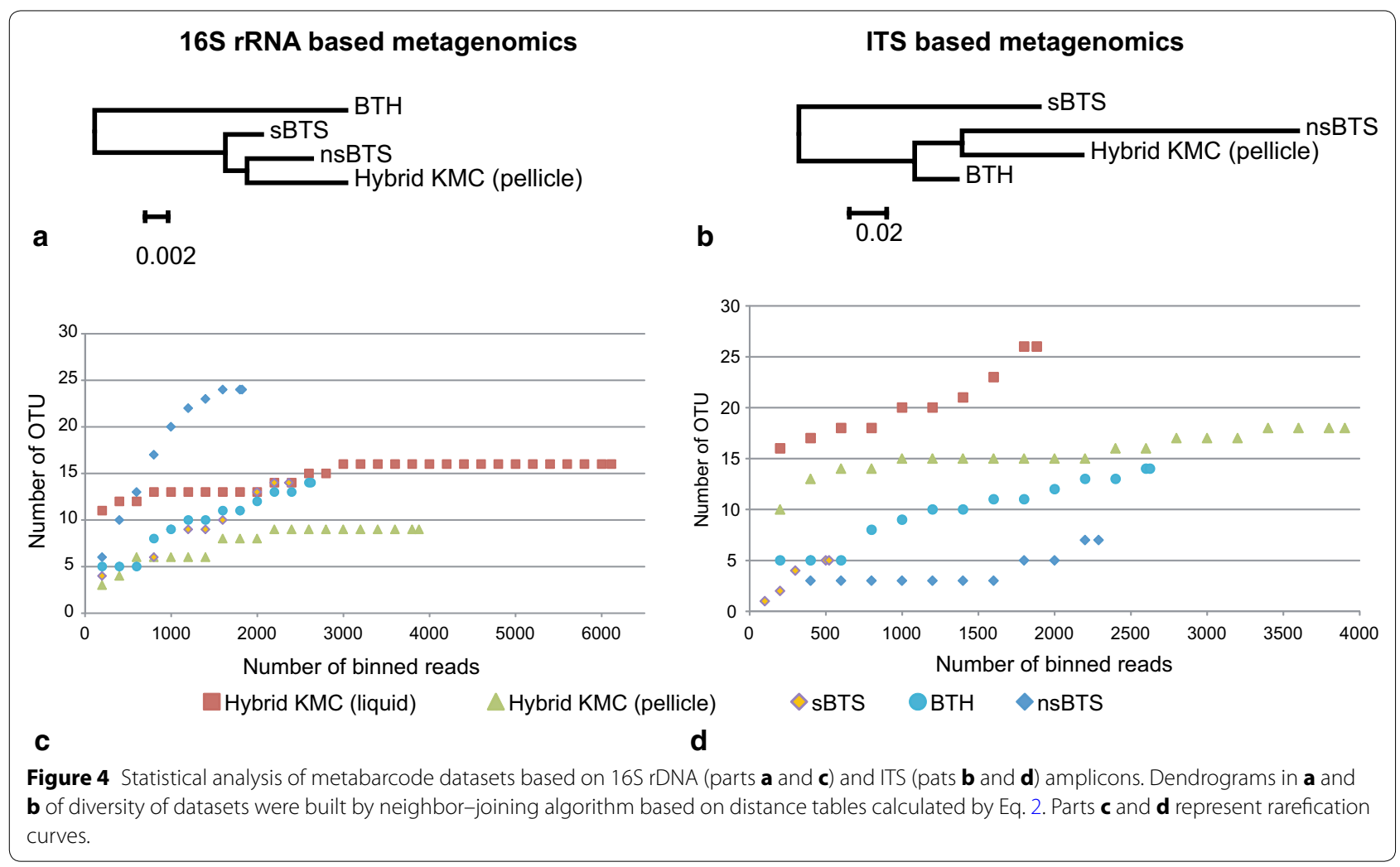

KMC-IMBG1 that both fungal and bacterial micro-flora of pellicle was more stable and less rich in different species than that from the liquid phase. Further research is needed to uncover the role of the core and accessory members of $\mathrm{KMC}$, including the uncultivable bacteria, and how they contribute to stabilizing the community and gaining its biologically active. The ability to modify $\mathrm{KMC}$ is of practical importance as a possible approach to improve the medicinal and biotechnological properties of the kombucha products (Kozyrovska et al. 2012). This work is the promising first step to design efficient and safe probiotics and synbiotics based on synthetic KMC communities of beneficial and harmless microbial species. It was hypothesized that the positive activity of kombucha probiotic on human health may be improved and extended by 'domestication' in the kombucha of other probiotic bacteria, e.g., lactobacilli, which in the current study most likely were recruited by KMC from the cabbage brine. There is still much to be discovered about bacteria-yeast communal interrelationships and their impact on the human microbiota. It also may be concluded that the DNA metabarcoding based on NGS is the best choice for profiling of complex microbial communities of fermented products.

\section{Additional files}

Additional file 1: Figure S1. Confocal scanning laser microscopy images of a cross section of cellulose-based pellicle produced by KMC in a sugared black tea, showing a variety of both bacteria and yeast cell morphotypes (a); cells of unusual morphology (a long shape), which may indicate the existence of dormant uncultivable microbial (sub)populations (b). Cellulose and yeast cells stained with calcofluor (a blue signal), bacterial cells and proteins stained with thiazine red (a yellow signal). Scale bar is $10 \mu \mathrm{m}$.

\section{Abbreviations}

KMC: kombucha microbial community; KMC-IMBG1: kombucha microbial community variant from Ukrainian collection; NGS: next generation sequencing; ITS: internal transcribed spacer; sBTS: kombucha culture grown on sterile black tea with sucrose; nsBTS: kombucha culture grown on non-sterile black tea with sucrose; BTH: kombucha culture grown on sterile black tea with honey.

\section{Authors' contributions}

$\mathrm{RO}$ performed the bioinformatics analysis and helped to draft the manuscript; ZI coordinated the study, helped to draft the manuscript and designed figures; OL carried out the molecular genetic studies; KO isolated bacterial and yeast strains from the kombucha culture; SS carried out the CSLM observations; PO performed the sequence alignment; VJ-P participated in the design of the study; KN conceived the study, participated in its design, drafted the manuscript. All authors read and approved the final manuscript. 


\section{Author details}

${ }^{1}$ Bioinformatics and Computational Biology Unit, Department of Biochemistry, University of Pretoria, Lynnwood road, Hillcrest, Pretoria 0002, South Africa. ${ }^{2}$ Institute of Molecular Biology and Genetics of National Academy of Sciences of Ukraine, Acad. Zabolotnoho str., 150, Kiev 03680, Ukraine. ${ }^{3}$ Institute of Planetary Science, DLR, Rutherfordstr. 2, 12489 Berlin, Germany.

\section{Acknowledgements}

We thank Dr. Olha Yaneva (Institute of Microbiology and Virology of National Academy of Sciences, Kyiv) for consultations in yeast isolation and culturing. This study has been partially supported by the grant of National Academy of Sciences of Ukraine (N47/2013)

\section{Compliance with ethical guidelines}

\section{Competing interests}

The authors declare that they have no competing interests.

Received: 26 May 2015 Accepted: 28 May 2015

Published online: 11 June 2015

\section{References}

Aloulou A, Hamden K, Elloumi D, Ali MB, Hargafi K, Jaouadi B et al (2012) Hypoglycemic and antilipidemic properties of kombucha tea in alloxaninduced diabetic rats. BMC Complement Alternat Med 16:12-63

Baldani JI, Caruso L, Baldani VLD, Goi SR, Döbereiner J (1997) Recent advances in BNF with nonlegume plants. Soil Biol Biochem 29:911-922

Battikh H, Bakhrouf A, Ammar E (2012) Antimicrobial effect of kombucha analogues. LWT Food Sci Technol 47:71-77

Bhattacharya S, Manna P, Gachhui R, Sil PC (2011) Protective effect of kombucha tea against tertiary butyl hydroperoxide induced cytotoxicity and cell death in murine hepatocytes. Indian J Exp Biol 49:511-524

Bhattacharya S, Gachhui R, Sil PC (2013) Effect of kombucha, a fermented black tea in attenuating oxidative stress mediated tissue damage in alloxan induced diabetic rats. Food Chemical Toxicol 60:328-340

Chao A (1984) Nonparametric estimation of the number of classes in a population. Scand J Stat 11:265-270

Gabor EM, de Vries EJ, Janssen DB (2003) Efficient recovery of environmental DNA for expression cloning by indirect extraction methods. FEMS Microbiol Ecol 44:153-163

Gupta S, Abu-Ghannam N (2012) Probiotic fermentation of plant based products: possibilities and opportunities. Crit Rev Food Sci Nutr 52:183-199

Hestrin S, Schramm M (1954) Synthesis of cellulose by Acetobacter xylinum 2 preparation of freeze-dried cells capable of polymerizing glucose to cellulose. Biochem J 58:345-352

Huson DH, Mitra S, Ruscheweyh H-J, Weber N, Schuster SC (2011) Integrative analysis of environmental sequences using MEGAN4. Genome Res 21:1552-1560

Illeghems K, De Vuyst L, Papalexandratou Z, Weckx S (2012) Phylogenetic analysis of a spontaneous cocoa bean fermentation metagenome reveals new insights into its bacterial and fungal community diversity. PLoS One 7:e38040

Jayabalan R, Malini K, Sathishkumar M, Swaminathan K, Yun S-E (2010) Biochemical characteristics of tea fungus produced during kombucha fermentation. Food Sci Biotechnol 19:843-847

Jung J, Lee SH, Kim JM, Park MS, Bae J-W, Hahn Y et al (2011) Metagenomic analysis of kimchi, a traditional Korean fermented food. Appl Environ Microbiol 77:2264-2274

Kallel L, Desseaux V, Hamdi M, Stocker P, Ajandouz EH (2012) Insights into the fermentation biochemistry of kombucha teas and potential impacts of kombucha drinking on starch digestion. Food Res Internat 49:226-232

Kim YS, Kim MC, Kwon SW, Kim SJ, Park IC, Ka JO et al (2011) Analyses of bacterial communities in meju, a Korean traditional fermented soybean bricks, by cultivation-based and pyrosequencing methods. J Microbiol 49:340-348

Kozyrovska N, Reva O, Goginyan V, de Vera J-P (2012) Kombucha microbiome as a probiotic: a view from the perspective of post-genomics and synthetic ecology. Biopolym Cell 28:103-110
Kurtzman CP, Robnett CJ (1997) Identification of clinically important ascomycetous yeasts based on nucleotide divergence in the $5^{\prime}$ end of the largesubunit (26S) ribosomal DNA gene. J Clin Microbiol 35:1216-1223

Lane DJ (1991) 16S/23S rRNA sequencing. In: Stackebrandt E, Goodfellow M (eds) Nucleic acid techniques in bacterial systematics. Wiley, New York, pp 115-175

Mandal MD, Mandal S (2011) Honey: its medicinal property and antibacterial activity. Asian Pac J Trop Biomed 1:154-160

Marsh AJ, O'Sullivan O, Hill C, Ross RP, Cotter PD (2014) Sequence-based analysis of the bacterial and fungal compositions of multiple kombucha (tea fungus) samples. Food Microbiol 38:171-178

Miller JH (1972) Experiments in molecular genetics. Cold Spring Harbor Laboratory, Cold Spring Harbor, p 436

Muyzer G, De Waal EC, Uitterlinden AG (1993) Profiling of complex microbial populations by denaturing gradient gel electrophoresis analysis of polymerase chain reaction-amplified genes coding for 165 rRNA. Appl Environ Microbiol 59:695-700

Nam YD, Lee SY, Lim SI (2012a) Microbial community analysis of Korean soybean pastes by next-generation sequencing. Int J Food Microbiol 155:36-42

Nam YD, Park SL, Lim SI (2012b) Microbial composition of the Korean traditional food "kochujang" analyzed by a massive sequencing technique. J Food Sci 77:250-256

Ogino A, Koshikawa H, Nakahara T, Uchiyama H (2001) Succession of microbial communities during a biostimulation process as evaluated by DGGE and clone library analyses. J Appl Microbiol 91:625-635

Oguntoyinbo FA, Narbad A (2012) Molecular characterization of lactic acid bacteria and in situ amylase expression during traditional fermentation of cereal foods. Food Microbiol 31:254-262

Ovcharenko LP (2013) Metagenomic analysis of domesticated kombucha multi-microbial culture. Biopolym Cell 29 (special issue: p 16)

Park E-J, Chun J, Cha C, Park W-C, Jeon C, Bae J-W (2012) Bacterial community analysis during fermentation of ten representative kinds of kimchi with barcoded pyrosequencing. Food Microbiol 30:197-204

Prado FC, Parada J, Pandey A, Soccol CR (2008) Trends in non-dairy probiotic beverages. Food Res Int 41:111-123

Puspita ID, Kamagata Y, Tanaka M, Asano K, Nakatsu CH (2012) Are uncultivated bacteria really uncultivable? Microb Environ 27:356-366

Sanger F, Nicklen S, Coulson AR (1977) DNA sequencing with chain-terminating inhibitors. Proc Natl Acad Sci USA 74:5463-5467

Shade A (2011) The kombucha biofilm: a model system for microbial ecology. In: Final report on research conducted during the Microbial Diversity course. Marine Biological Laboratories, Woods Hole, MA

Shade A, Caporaso G, Handelsman J, Knight R, Fierer N (2013) A meta-analysis of changes in bacterial and archaeal communities with time. ISME J 7:1493-1506

Srihari T, Karthikesan K, Ashokkumar N, Satyanarayana U (2013) Antihyperglycaemic efficacy of kombucha in streptozotocin-induced rats. J Funct Foods 5:1794-1802

Tamura K, Stecher G, Peterson D, Filipski A, Kumar S (2013) MEGA6: molecular evolutionary genetics analysis version 6.0. Mol Biol Evol 30:2725-2729

Teoh AL, Heard G, Cox J (2004) Yeast ecology of kombucha fermentation. Int J Food Microbiol 95(2):119-126

Vasudha S, Mishra HN (2013) Non-dairy probiotic beverages. Int Food Res J 20:7-15

White TJ, Bruns T, Lee S, Taylor J (1990) Amplification and direct sequencing of fungal ribosomal RNA genes for phylogenetics. In: Innis MA, Gelfand DH, Sninsky JJ, White TJ (eds) PCR protocols: a guide to methods and applications. Academic Press, New York, pp 315-322

Wright ES, Yilmaz LS, Noguera DR (2012) DECIPHER, a search-based approach to chimera identification for $16 \mathrm{~S}$ rRNA sequences. Appl Environ Microbiol 78:717-725

Yamada Y, Yukphan P, Lan Vu HT, MuramatsuY Ochaikul D, Tanasupawat S et al (2012) Description of Komagataeibacter gen nov with proposals of new combinations (Acetobacteraceae). J Gen Appl Microbiol 58:397-404

Yapar K, Cavusoglu K, Oruc E, Yalcin E (2010) Protective effect of kombucha mushroom (KM) tea on phenol-induced cytotoxicity in albino mice. J Environ Biol 31:615-621 